\title{
Energy Efficient Stable Election Protocol Scheme for Extend the Lifetime of WSN with Isolated Nodes
}

\author{
Sonam Verma \\ M.Tech (CSE) \\ AISECT UNIVERSITY \\ Bhopal, (M.P)
}

\author{
Ayonija Pathre \\ ASSIST PROF \\ AISECT UNIVERSITY \\ Bhopal, (M.P)
}

\begin{abstract}
We propose Sep, a heterogeneous-aware protocol to prolong the time interval before the death of the primary node (we visit as stability period), that is crucial for several applications wherever the feedback from the device network should be reliable. Sep is predicated on weighted election possibilities every node to become cluster head per the remaining energy in each node. We tend to show by simulation that Sep always prolongs the soundness amount compared to (and that the typical outturn is bigger than) the one obtained using current bunch protocols. We tend to conclude by learning the sensitivity of our Sep protocol to heterogeneity parameters capturing energy imbalance within the network. We tend to found that Sep yields longer stability region for higher values of additional energy brought by additional powerful nodes.
\end{abstract}

\section{Keywords}

Energy aware clustering, energy consumption, distributed clustering, isolated nodes.

\section{INTRODUCTION}

Energy efficiency is one in every of the most necessary design goals for wireless sensor networks (WSNs). To the current effect, clustering is generally used to prolong the period of WSNs. during this study, we tend to propose a new energy efficient (EE) clustering primarily based protocol for single-hop, heterogeneous WSNs. The projected protocol uses channel state information (CSI) within the selection method of Cluster Heads (CHs). It's shown through simulations in MATLAB that the projected protocol has 1.62 to 1.89 times higher stability amount than that of a well known protocols as well as LEACH, DEEC, and SEP.

Wireless sensor network has appeared as an important space for analysis and development. The key constraints within the development of wireless sensor networks (WSN) are value, restricted energy and memory, restricted process capability, and also the memory size of the sensor nodes. WSN are operating as long as they will communicate sensed information to manage particular node. Sensing and communication are essential activities and that they consume energy thus power executive and coverage preservation will effectively network period increase. In some protocols sensor nodes transmit their information on to a BS. The most necessary feature of a routing protocol is reduced energy consumption and extension of the network's period. Throughout the recent years, additional energy efficient routing protocols are projected for WSNs.

The technology for sensing and manage includes electrical and field sensors, radio-wave frequency sensors. Sensor is usually deployed in a very high-density manner and in giant quantities. one amongst the part of wireless sensor network an assembly of calculated or localized sensors, an interconnecting network, a central purpose of data cluster and a collection of computing sources at the central purpose to handle data correlation, event trending, standing querying, and data mining.

Wireless sensor Networks (WSN) is a rising technology within the Wireless Network field that is consists of little nodes. These sensor nodes are self - energized and have low computation power. Battery is that the primary power supply during a sensor node [1]. A sensor node carries limited and usually irreplaceable power sources. Therefore to realize top quality of service provisions, sensor network conventions should concentrate basically on power conservation [2]. Cluster sensor nodes could be a productive topology control technique to reduce energy consumption of the sensor nodes for increasing time period of WSNs [3]. Cluster the nodes has several benefits, like quantifiability, energy efficiency and reducing routing delay [4]. Cluster primarily based routing involves the creation of clusters, election of cluster heads and routing through the Cluster Heads $(\mathrm{CH})$. The energy is preserved a lot of by the $\mathrm{CH}$ by collecting the information within the cluster, pressure it so transmission the collective information to the base station [6]. The 2 imperative steps in cluster theme are $\mathrm{CH}$ determination and cluster formation [5]. Improperly designed cluster algorithms will cause nodes to become isolated from $\mathrm{CHs}$ as shown in Fig. 1. Communication of those isolated nodes with the sink can consume a lot of energy. If the sink is much away from these isolated nodes, then isolated nodes cannot directly communicate with the sink. If these isolated nodes are not within the vary of near current cluster heads, then isolated nodes cannot communicate with this cluster head additionally. The authors in Regional Energy Aware cluster with Isolated Nodes (REAC-IN) thought of the regional average energy and also the distance from sensors to the sink and it confirm whether or not the isolated node sent its information to a $\mathrm{CH}$ node within the previous round or to the sink. The drawback during this paper is that if the previous round $\mathrm{CH}$ is much away than the other neighbor node of the isolated nodes, then it consumes energy for transmitting the message to the sink. Moreover, in their work, the distance from the isolated node to sink through relay node was thought of. however if the relay node is $\mathrm{CH}$ of previous spherical, then another time $\mathrm{CH}$ has got to transmit the information to its current $\mathrm{CH}$ that they need not considered.

\section{CLUSTERING}

Clustering have witnessed in improvement of network life time and effective approach to reduce energy consumption of nodes. Clustering of nodes avoid long distance communication of nodes to BS. Only few nodes i.e. $\mathrm{CHs}$ are causing data over long distance. Avoidance of long distance communication is preserving energy of sensor nodes. Whereas reduction of data due to aggregation conserves energy of $\mathrm{CHs}$. 
clustering schemes use TDMA schedule for intra cluster communication. Nodes are appointed slot for sending data. Nodes conserve energy by transiting to sleep state for slots of various nodes and avoid idle listening and overhearing. Nodes are causing data with slot assigned to them, so avoid collision. Avoiding collision, idle listening, and overhearing additional conserves energy of nodes.

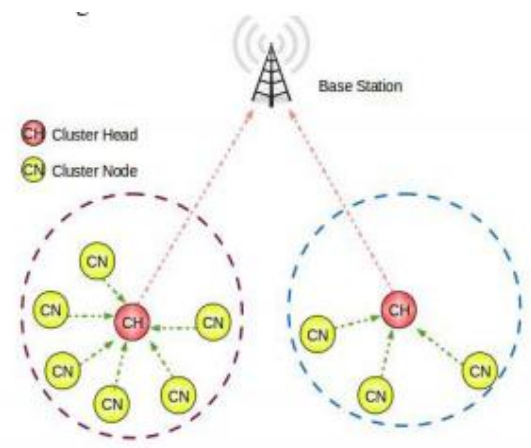

Fig.1 Cluster based wireless sensor network

In cluster-based WSN, sensors are organized in clusters each having one sensor promoted as $\mathrm{CH}$. All non- $\mathrm{CH}$ nodes transmit their data to their $\mathrm{CH}$ that routes it to the remote PN. Clustering can provide for substantial energy saving since only $\mathrm{CH}$ sensors are involved in routing and relaying data. Some necessary factors for portal kind of WSN as follows:

- Connectivity and coverage

- Data aggregation

- Node deployment and transmission

- Energy utilization

- $\quad$ Fault tolerance

- Reliable performance with QoS

- Network dynamics with scalability

- Data sensing and report modeling

Main forms of clustering routing protocols may be classified into following categories:

- Data central protocols

- Hierarchical protocols

- Location primarily based protocols

- Network flow based and QoS aware protocols

\section{STABLE ELECTION PROTOCOL (SEP)}

The Stable Election Protocol (SEP) could be a hierarchical sensor network protocol. The Sep rule has been enforced with a number of the sensor nodes within the network equipped with higher energy levels [9]. Higher energy state nodes are referred to as advanced nodes in Sep [1]. Sep provides stability during a network; however the network lifetime decreases rapidly once the death of the primary sensor node. During a little network, LEACH provides network stability, however sep offers higher performance than LEACH.

In Stable Election Protocol (SEP) (Smaragdakis, Matta, and Bestavros), the authors have shown that the classical protocol like LEACH cannot benefit of network heterogeneousness. Network heterogeneousness is earned by assignment additional energy to the fraction of total nodes. These nodes are known as as advanced nodes, whereas the remaining nodes are the traditional nodes. The study of optimal chance of being cluster head as a perform of spatial density, has been studied through simulations (Heinzelman, Chandrakasan, and Balakrishnan, "Energy efficient communication protocol for wireless small sensor networks") or analytically (Bandyopadhyay and Coyle, "An energy efficient hierarchical clustering rule for wireless sensor networks" "Minimizing communication prices in hierarchically clustered networks of wireless sensors") below the constraint that nodes are uniformly distributed over the sensor field. Such clustering scheme is perfect once energy consumption is well distributed over all sensors and total energy consumption is minimum.

In SEP, a node assumes a random number between 0 and 1 and a node is elected as $\mathrm{CH}$, if the selected random number is less than the threshold. As SEP uses the heterogeneous network, threshold values are different for normal nodes and advanced nodes. The threshold value for normal nodes is given by

$$
T\left(S_{n r m}\right)= \begin{cases}\frac{P_{n r m}}{1-p n r m *\left(r \bmod \frac{1}{p n r m}\right)} & \text { if } S_{n r m} \in G^{\prime} \\ 0 & \text { otherwise }\end{cases}
$$

Where $r$ is the current round, G' is the set of nodes that have not become Cluster Heads within the last $1 /$ pnrm rounds, $\mathrm{T}(\mathrm{Snrm})$ is the threshold applied to a population of $\mathrm{N}$ nodes, Pnrm is the probability of the normal nodes to become Cluster Heads, given by

$$
\text { Pnrm }=\frac{\text { Popt }}{1+\alpha * m}
$$

Where $\mathrm{m}$ is the percentage of total nodes, which are advanced nodes, $\alpha$ is the percentage of more energy given to the advanced nodes, and Popt is given by

$$
\text { Popt }=\frac{k_{o p t}}{N}
$$

Where $\mathrm{N}$ is the total number of nodes in the network and kopt is the optimal number of Cluster Heads. The threshold value for advanced nodes is given by

$$
T\left(S_{a d v}\right)= \begin{cases}\frac{P_{a d v}}{1-P_{a d v} *\left(r \bmod \frac{1}{P_{a d v}}\right)} & \text { if } S_{a d v} \in G^{\prime \prime} \\ 0 & \text { otherwise }\end{cases}
$$

Where $r$ is the current round, G', is the set of nodes that have not become Cluster Heads within the last 1/ Padv rounds, $\mathrm{T}(\mathrm{Sadv})$ is the threshold applied to a population of $\mathrm{N} * \mathrm{~m}$ nodes, Padv is the probability of the advanced nodes to become Cluster Heads and it is given by

$$
P_{a d v}=\frac{P_{o p t}}{1+\alpha * m} *(1+\alpha)
$$

In authors have proposed Low Energy Adaptive Clustering Hierarchy with deterministic cluster head selection by introducing new parameters for electing a cluster head: the remaining energy and its meaningful variance? Nodes with the highest remaining energy and lower energy variance are 
having more probability to become $\mathrm{CHs}$. A node participating in the $\mathrm{CH}$ election process generates a random number between 0 and 1 , which is compared with the threshold value $\mathrm{T}(\mathrm{n})$, given by

$$
T(n)=\left\{\begin{array}{cc}
\frac{p}{1-p *\left(r \bmod \frac{1}{p}\right)} * \frac{\gamma_{i}(r) * E_{i}(r)}{E_{o}} & \text { if } n \in G \\
& \text { otherwise }
\end{array}\right.
$$

$\mu_{i}(r)=\frac{1}{r} \sum_{r=1}^{r} E_{i}(r)$

Where Ei(r) is the node energy during round r, Eo is the initial energy of the nodes, $\mathrm{p}$ is the desired percentage of $\mathrm{CH}$ given by Equation 4.7and $\gamma \mathrm{i}(\mathrm{r})$ is the convex function to ensure convergence of the algorithm given by Equation 4.9.

$$
P=\frac{K_{o p t}}{n}=\frac{\sqrt{\frac{n}{2 * \pi}} * d_{0} * \frac{M}{d_{t o B S}^{2}}}{n}
$$

Here, $\mathrm{n}$ is the number of nodes deployed in a square region of M x M meter ${ }^{2}, d_{0}=\sqrt{\frac{\epsilon_{f s}}{\epsilon_{m p}}}, \in_{f s}$ and $\epsilon_{m p}$ depend on the transmitter amplifier models used for the experiments and $d_{t o B S}$ is the distance between cluster head and the BS given by

$$
\begin{aligned}
& d_{t o B S}=0.765 * \frac{M}{2} \\
& \gamma_{i}(r)=\frac{\mu_{i}(r)}{\sqrt{\mu_{i}^{2}(r)+v_{i}(r)}}
\end{aligned}
$$

In Equation 9, vi(r) represents the variance of the energy level and $\mu \mathrm{i}(\mathrm{r})$ represents the average energy of a node at round $\mathrm{r}$. $\operatorname{vi}(\mathrm{r})$ and $\mu \mathrm{i}(\mathrm{r})$ is given by Equations 10 and 11 respectively.

$$
v_{i}(r)=\frac{1}{r} \sum_{r=1}^{r}\left(E_{i}(r)-\mu_{i}(r)\right)^{2}
$$

In this section we describe SEP, which improves the stable region of the clustering hierarchy process using the characteristic parameters of heterogeneity, namely the fraction of advanced nodes (m) and the additional energy factor between advanced and normal nodes $(\alpha)$

\section{RESULTS}

In this section, simulation results of our proposed algorithm and discussed under different network sizes with respect to performance metrics i.e. energy consumption and consumption delay.

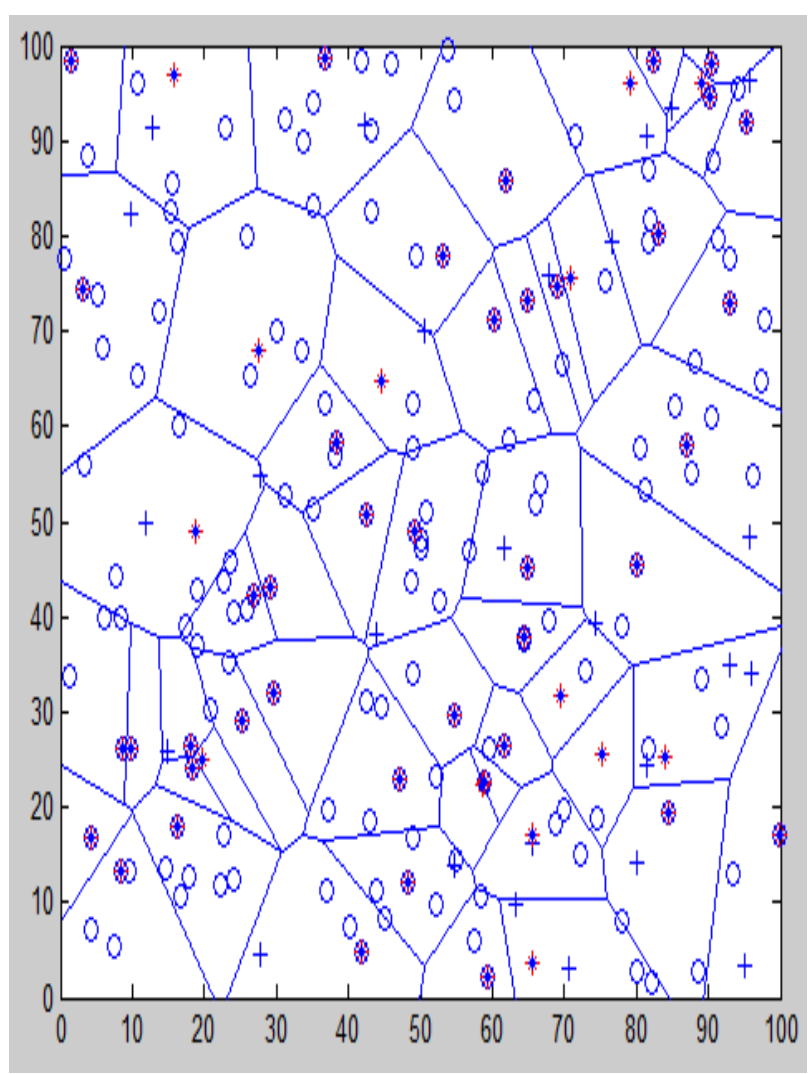

Fig.1 Cluster Head in the WSN

Figure 1 shows the cluster head in the wireless sensor network. The cluster heads can be selected randomly or based on one or more criteria. Selection of cluster head largely affects WSNs lifetime. Ideal cluster head is the one which has the highest residual energy.

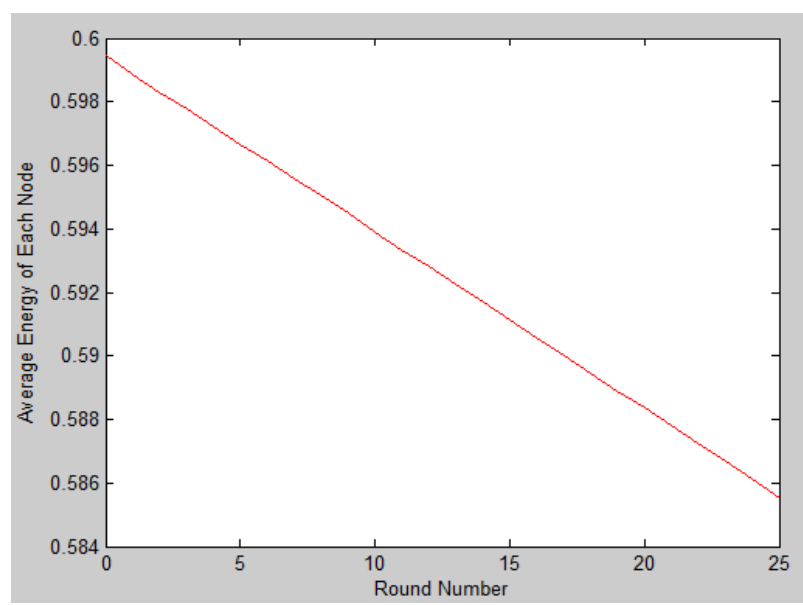

Fig.2 Average Energy of EACH Node v/s Round Number

Fig. 2 shows average energy of each node v/s round number. In this figure $\mathrm{x}$ axis show round number and $\mathrm{y}$ axis show average energy of each node. 


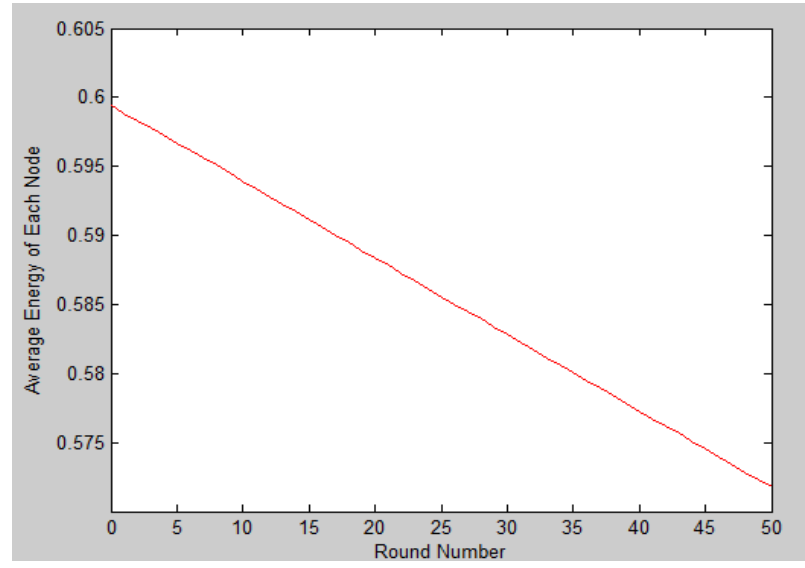

Fig.3 Average Energy of each Node v/s Round Number

Fig. 3 shows average energy of each node v/s round number. In this figure $\mathrm{x}$ axis show the round number and $\mathrm{y}$ axis show average energy of each node.

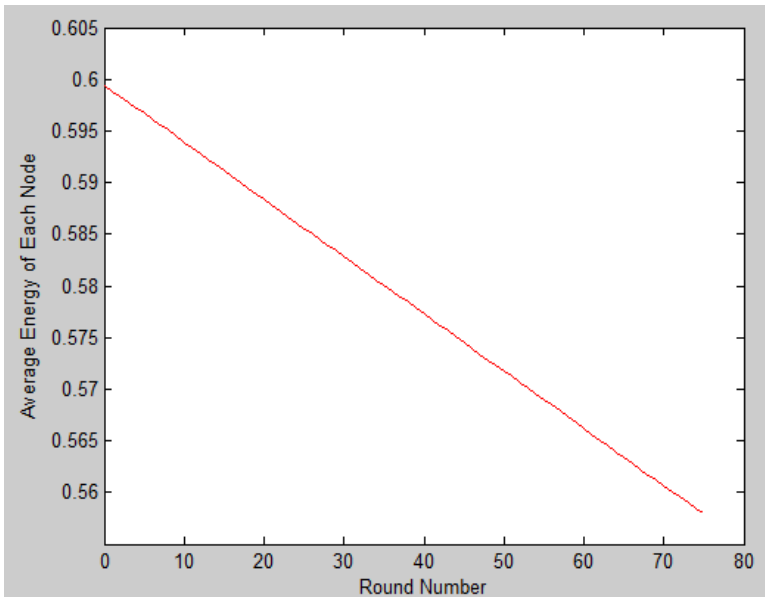

Fig.4Average Energy of Each Node v/s Round Number

Fig.4 shows average energy of each node v/s round number. In this figure $\mathrm{x}$ axis show round number and $\mathrm{y}$ axis show average energy of each node.

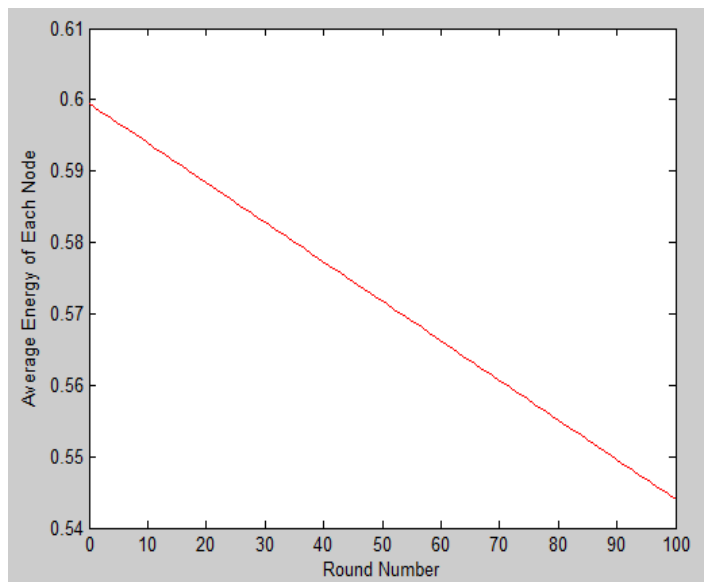

Fig.5 Average Energy of Each Node v/s Round Number

Fig. 5 shows average energy of each node v/s round number. In this figure $\mathrm{x}$ axis show round number and $\mathrm{y}$ axis show average energy of each node.

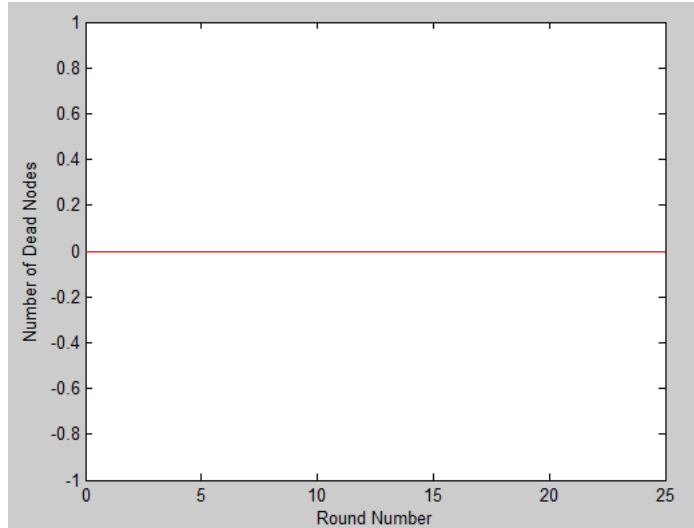

Fig.6 Number of Dead Nodes v/s Round Number

Fig. 6 show the number of dead nodes v/s number of round. In this figure $\mathrm{x}$ axis represent the round number and $\mathrm{y}$ axis represent the number of dead nodes.

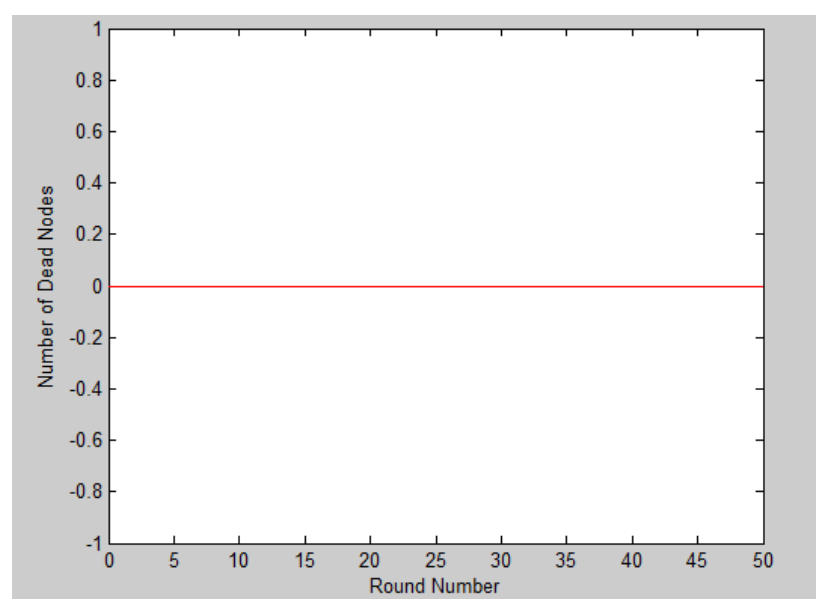

Fig.7 Number of Dead Nodes v/s Round Number

Fig. 7 show the number of dead nodes v/s number of round. In this figure $\mathrm{x}$ axis represent the round number and $\mathrm{y}$ axis represent the number of dead nodes

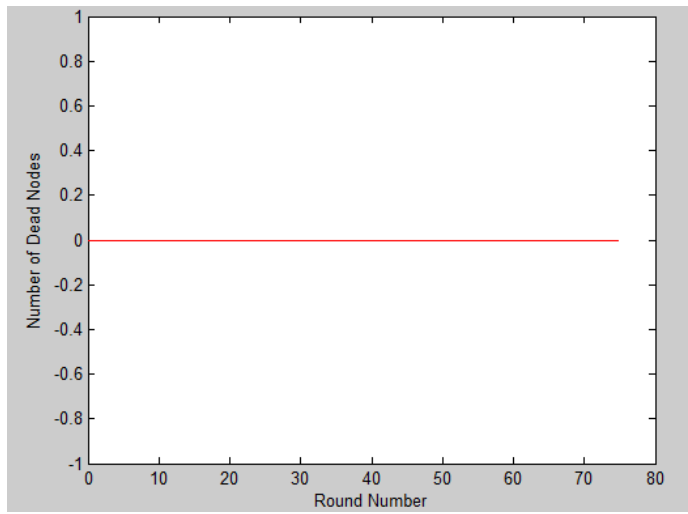

Fig.8 Number of Dead Nodes v/s Round Number

Fig. 8 show the number of dead nodes v/s number of round. In this figure $\mathrm{x}$ axis represent the round number and $\mathrm{y}$ axis represent the number of dead nodes. 


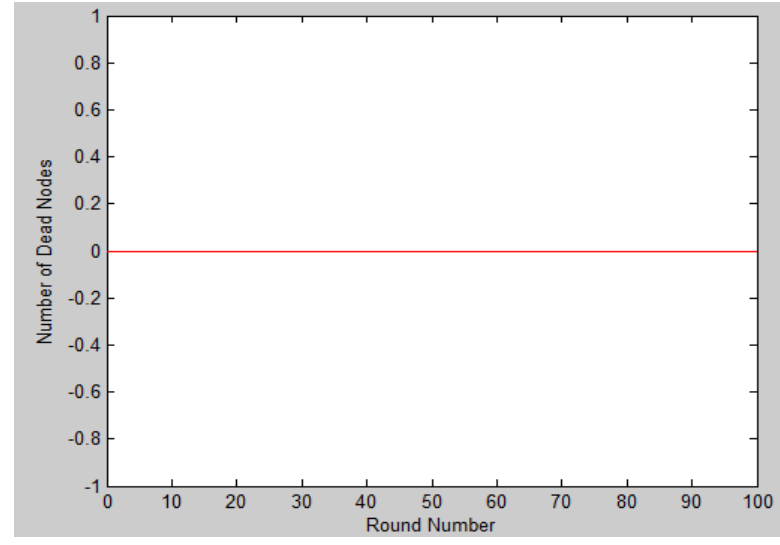

Fig.9 Number of Dead Nodes v/s Round Number

Fig. 9 show the number of dead nodes v/s number of round. In this figure $\mathrm{x}$ axis represent the round number and $\mathrm{y}$ axis represent the number of dead nodes with different no. of Random number for node.

\section{CONCLUSION}

In this paper, by considering the benefits and drawbacks of our previous works, we tend to planned a replacement Energy efficient clustering algorithmic rule for Wireless detector Networks. It's the power of selecting a cluster heads of clusters of the network. Evaluated the performance of our planned scheme sep preparation and compared with LEACH protocol using MATLAB in several network situations, simulation experiments show that the planned scheme provides higher results than the opposite existing protocol with reference to energy consumption and consumption delay the longer term study can lie the way to choose cluster head from the chosen cluster heads of cluster and try to consume additional energy with less delay.

\section{REFERENCES}

[1] Leu, Jenq-Shiou, et al. "Energy efficient clustering scheme for prolonging the lifetime of wireless sensor network with isolated nodes." IEEE communications letters 19.2 (2015): 259-262.
[2] Li, Bin, et al. "Energy-effective relay selection by utilizing spacial diversity for random wireless sensor networks." IEEE Communications Letters 17.10 (2013): $1972-1975$

[3] Ritu Kadyan, Kamal Saluja "Distributed Energy Efficient Clustering (DEEF) in Heterogeneous Wireless Sensor Networks", International Journal of Engineering and Innovative Technology (IJEIT) Volume 4, Issue 1, July 2014.

[4] Younis, Ossama, and Sonia Fahmy. "HEED: a hybrid, energy-efficient, distributed clustering approach for ad hoc sensor networks." IEEE Transactions on mobile computing 3.4 (2004): 366-379.

[5] Heinzelman, Wendi Rabiner, Anantha Chandrakasan, and Hari Balakrishnan. "Energy-efficient communication protocol for wireless microsensor networks." System sciences, 2000. Proceedings of the 33rd annual Hawaii international conference on. IEEE, 2000.

[6] Chaubey, Nirbhay K., and Dharti H. Patel. "Energy Efficient Clustering Algorithm for Decreasing Energy Consumption and Delay in Wireless Sensor Networks (WSN)." Energy 4.5 (2016)

[7] Chaubey, Nirbhay K., and Dharti H. Patel. "Energy Efficient Clustering Algorithm for Decreasing Energy Consumption and Delay in Wireless Sensor Networks (WSN)." Energy 4.5 (2016).

[8] Singh, Jagdeep, and Manju Bala. "Varsha," REAC-IN Regional Energy Aware Clustering Protocol in Wireless Sensor Network." International Journal of Computer Sciences and Engineering 4: 90-94.

[9] Logambigai, R., S. Ganapathy, and A. Kannan. "Cluster based routing with isolated nodes in WSN." International Journal for Research in Applied Science \& Engineering Technology (IJRASET) 4.3 (2016).

[10] Thamizhamuthan, N., and T. Prabakaran. "NETWORK LIFETIME ENRICHMENT PROTOCOL FOR WIRELESS SENSOR NETWORKS USING EESEP PROTOCOL." (2015) 